\title{
NOTICIARIO
}

\section{PALÉOENVIRONMENT DURANT LE PLÉISTOCENE SUPÉRIEUR EN VAUCLUSE: ANALYSE PALYNOLOGIQUE DES COUCHES INFÉRIEURES DE L'ABRI DE LA COMBETTE (BONNIEUX, VAUCLUSE, FRANCE)}

\author{
PALEOAMBIENTE DURANTE EL PLEISTOCENO \\ SUPERIOR EN VAUCLUSE: ANÁLISIS \\ PALINOLÓGICO DE LOS NIVELES \\ INFERIORES DEL ABRIGO DE LA COMBETTE \\ (BONNIEUX, VAUCLUSE, FRANCIA)
}

PALEOENVIRONMENT DURING THE UPPER

PLEISTOCENE AT VAUCLUSE: PALYNOLOGICAL

ANALYSIS OF THE LOWER LAYERS OF L'ABRI

DE LA COMBETTE (BONNIEUX, VAUCLUSE, FRANCE)

\author{
JOSÉ ANTONIO LÓPEZ-SÁEZ (*) \\ PIERRE JEAN TEXIER (**) \\ BUI-THI-MAI $(* * *)$
}

\section{RESUMÉ}

L'étude palynologique de l'abri de La Combette nous informe sur l'évolution de la végétation autour du site pendant le Pléistocène supérieur, au Würm ancien (début du stade isotopique 3). Les différentes phases climatiques mises en évidence sont caractérisées par la dominance du pin sylvestre et la présence notable d'espèces méditerranéennes. On constate le caractère refuge du site.

\section{RESUMEN}

El estudio palinológico delAbrigo de La Combette nos informa sobre la evolución seguida por la vegetación en el

(*) Laboratorio de Arqueobotánica. C.E.H. CSIC. Duque de Medinaceli 8. 28014 Madrid. Espagne.

(**) Préhistoire et Technologie. C.R.A. C.N.R.S. 250 rue Albert Einstein. Valbonne. 06560 Sophia Antipolis. France.

(***) Laboratoire de Palynologie. C.R.A. C.N.R.S. 250 rue Albert Einstein. Valbonne. 06560 Sophia Antipolis. France.

El artículo fue remitido en su versión final el 28-V-98. entorno del yacimiento durante el Pleistoceno Superior, Würm antiguo (inicio del estadio isotópico 3). Se ponen de manifiesto diversas fases climáticas caracterizadas, en general, por el dominio de Pinus sylvestris t. así como por la notable presencia de especies mediterráneas. Se constata el cáracter de refugio de la zona estudiada.

\begin{abstract}
The palynological study of the La Combette rockshelter gives us information on the evolution of the vegetation surrounding the rock shelter area during the Upper Pleistocene (isotopic stage 3). Although widely dominated by Pinus sylvestris t., mediterranean species are also present in the different climatic phases revealed by the analysis. This confirms the refuge character of the area.
\end{abstract}

Mots-clés: Palynologie. Paléolithique Moyen. Moustérien. Pléistocène Supérieur. Stade isotopique 3. Vaucluse. France. 
Palabras clave: Palinología. Paleolítico Medio. Musteriense. Pleistoceno Superior. Estadio isotópico 3. Vaucluse. Francia.

Keywords: Palynology. Middle Palaeolithic. Mousterian. Upper Pleistocene. Isotopic stage 3. Vaucluse. France.

\section{INTRODUCTION}

L'abri sous roche moustérien du pont de La Combette (Commune de Bonnieux, Vaucluse) a été découvert en 1971 par J. Brochier et M. Livache lors d'une campagne de prospection géologique (Brochier, 1976). Les fouilles de son épais remplissage ont été lancées en 1986 parAndré Tavoso et se poursuivent actuellement sous la direction de Pierre-Jean Texier (1). Plusieurs publications ont déjà été consacrées à l'exploitation scientifique du site (Brochier, 1977; Buisson-Catil, 1994, 1997; Lemorini 1997; Thery 1998; Texier et alii, 1998a, b).

De nombreux éléments convergent pour attribuer la partie supérieur du remplissage de l'abri au début du stade isotopique 3 (Abassi et Desclaux, 1996; Richter et alii, 1996) (2). Une date moyenne TL sur loess de 57,4 +/- 4,3 ka est proposée pour les limons supérieurs (3).

Une première analyse palynologique des dépôts archéologiques a été réalisée par M. Bui-ThiMai (1) dans les limons supérieurs et moyens, ainsi que dans le foyer E de l'ensemble sédimentaire inférieur. Les tests réalisés par cette dernière (cotes 156 à $-495 \mathrm{~cm}$ ), ont malheureusement donné des résultats assez décevants en raison du mauvais état de conservation du contenu sporo-pollinique du sédiment dans cette partie du remplissage de l'abri. Parmi les pollens identifiés on notera la présence remarquable d'Olea dans un échantillon prélevé à la cote $-484 \mathrm{~cm}$, ainsi que la dominance de Pinus dans les échantillons prélevés entre -484 et $-495 \mathrm{~cm}$ ainsi que la présence en proportions notables dans le même niveau, d'espèces indicatrices d'une anthropisation (Cichorioideae, Anthemis t.). Les échantillons prélevés dans les niveaux supérieurs $(-156 /-160,-177,5,-218 /-222,-275$ et $-325 \mathrm{~cm})$ sont trop pauvres, pour permettre d'aboutir à des conclusions d'ordre paléoécologique. En revanche,

(1) Texier, P.J. (1994): L'abri de La Combette, site paléolitique moyen à Bonieux (Vaucluse). Fouille programmée, rapport de synthèse 1989-1994. Valbonne. 69 pp.

(2) Heléne Valladas (communication orale). Laboratoire des Sciences du Climat et de l'Environment: Gif-sur-Yvette. France.

(3) G. Wagner (communication orale). Max Plank Institüt für Kernphysik. Heidelberg. Allemagne. la fouille a permis de recueillir une quantité assez importante de charbons de bois bien conservés. Nous disposons ainsi pour les mêmes niveaux sédimentaires, d'une étude anthracologique (Thery, 1998) mettant en évidence dans l'environnement proche, la présence de Salix, Pinus sylvestris/nigra, et d'un Rhamnaceae (couche B/C et D), de Pinus sylvestris/nigra, Buxus, Acer et Salix (Couche E).

Nous disposons également d'études sur la pluie pollinique actuelle pour les corylaies du Luberon (Triat, 1971).

Le Luberon est certainement la montagne la plus individualisée de ces chaînons orientés est-ouest, si caractéristiques de la Provence. Il marque entre Cavaillon et Manosque, la frontière entre la Haute et la Basse Provence. Son intérêt particulier réside essentiellement dans le fait qu'il est sans doute l'un des derniers massifs montagneux de Provence à conserver encore un équilibre écologique relativement satisfaisant (Guende, 1993).

La haute définition de l'information fournie par l'étude pluridisciplinaire des niveaux moustériens a permis, dans le cas le plus favorable (niveau D), d'évaluer la taille et la mobilité des groupes humains qui ont fréquenté l'abri du Pont de La Combette, d'estimer quels ont été leurs besoins de subsistance et d'apprécier leurs stratégies socio-culturelles (Texier et alii, 1998b). Ainsi est-il parfois possible d'aborder dans le détail, la fonction du site et son rôle dans le territoire (Texier et alii, 1998a). L'analyse palynologique constitue donc un apport important d'informations sur le climat du moment et sur son évolution. Elle apporte également des éléments nouveaux sur l'environnement lointain, voisin ou local (Geneste, 1989) de l'abri. Enfin, elle contribue avec l'anthracoanalyse, à enrichir notre information sur l'impact qu'ont éventuellement pu avoir les petits groupes de chasseurs-cueilleurs de La Combette sur leur environnement.

\section{SITUATION GÉOGRAPHIQUE ET CONTEXTE GÉOLOGIQUE}

Au coeur du Parc Naturel du Luberon, à trois kilomètres au sud-est de Bonnieux et à huit d'Apt, l'abri du pont de La Combette n'est, dans le vallon de La Combette, qu' à quelques centaines de mètres des gorges de l'Aiguebrun, qui incisent en cluse le massif et séparent ainsi le Petit du Grand Lubéron (Fig. 1).

Situé dans un vallon étroit à proximité de plusieurs sources pérennes qui jalonnent dans toute la ré- 


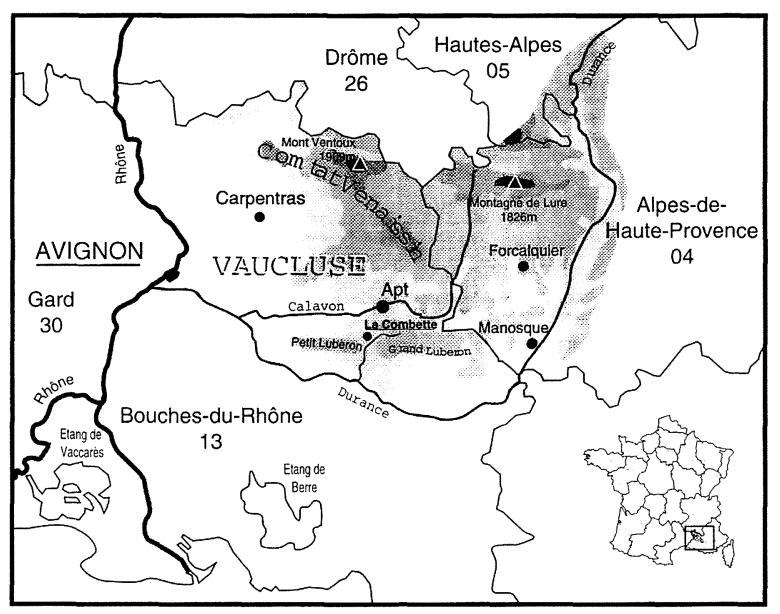

Fig. 1. Département de Vaucluse et situation de l'abri dans la partie méridionale du territoire parcouru par les moustériens de La Combette.

gion dans le contact Miocène-Crétacé, l'abri est particulièrement bien protégé des vents parfois très violents soufflant dans la région. Creusé au pied d'une falaise de molasse miocène (Burdigalien), il est orienté plein sud. Le site de La Combette se trouve à $327 \mathrm{~m}$ d'altitude, dans une zone déprimée de la chaîne montagneuse d'orientation ouest-est. Les séries calcaires crétacées se relèvent à l'ouest et à l'est pour culminer respectivement à 719 et $1125 \mathrm{~m}$.

L'abri du Pont de La Combette, comme les autres sites moustériens de la région, est partie intégrante d'un ensemble géologique bien délimité, encadré au nord part les versants méridionaux du mont Ventoux et de la montagne de Lure, à l'ouest par la bordure orientale du couloir rhodanien (plaines de Carpentras et de Cavaillon), au sud par le synclinal de la Basse Durance, d'axe est-ouest, et à l'est par l'accident médio-durancien d'orientation NNE-SSO. Des reliefs montagneux culminant au nord à près de $2000 \mathrm{~m}$ et deux puissants cours d'eau, le Rhône et la Durance, en constituaient les limites géographiques.

Le paysage est fortement contrasté. Des massifs calcaires secs, d'altitude élevée (Mt.Ventoux, Montagne de Lure) et des reliefs karstiques (Monts de Vaucluse, Plateaux d'Albion, de la Garde d'Apt, de St. Christol...) d'âge barrémien-bédoulien s'étageant de 600 à 1100 m, s'oppose notamment à des pentes plus douces et plus humides disséquées par l'érosion à travers les séries ocreuses violemment colorées de l'albien-cénomanien. Le Coulon-Calavon autour duquel est construit le réseau hydrographique, est contraint de suivre une direction con- séquente E-O par l'anticlinal du Luberon. Il emprunte alors l'axe du synclinal Forcalquier-Apt à hauteur de Céreste où il draine essentiellement des séries tertiaires.

\section{STRATIGRAPHIE}

Le remplissage connu de l'abri se développe sur environ 7 m d'épaisseur (Texier et alii, 1998b). Le processus de mise en place des sédiments (Fig. 2) peut s'interpréter globalement de la manière suivante:

- dépôt sur le fond rocheux d'un premier ensemble limoneux à granules (EL inf.), encore mal connu, renfermant les vestiges d'au moins une occupation moustérienne (couche F-H). Niveau très partiellement fouillé mais exploré par carottage jusqu'à ce qui pourrait être le fond rocheux de l'abri, à près de $7 \mathrm{~m}$ sous le niveau de référence.

- mise en place, en discordance de ravinement, d'un premier apport torrentiel à graviers, blocs et grandes dalles de molasse.

- dépôt de sédiments limoneux corrélativement à une importante occupation moustérienne (couche E: silex, vestiges osseux, charbons de bois, cendres...)

- mise en place relativement violente, d'un second apport torrentiel à graviers, blocs et dalles de molasse, ravinant la couche $\mathrm{E}$ et la tronquant dans la partie occidentale de l'abri.

- début de l'ensemble supérieur (EL sup.) marqué par un changement brutal de sédimentation: dépôt sub-horizontal des limons à blocs en discordance sédimentaire sur le cône torrentiel mis en place précédemment. Un niveau d'occupation moustérien sub-horizontal a pu y étre décapé sur une cinquantaine de $\mathrm{m}^{2}$ (couche $\mathrm{D}$ ).

- dépôt de limons lités, stériles

- dépôt sur plus de 2 m d'épaisseur, de limons passant progressivement à des loess francs. Deux niveaux d'occupation (B/C et $\mathrm{A})$, ont été reconnus dans cette formation.

Deux approches complémentaires sont actuellement poursuivies et visent par la géologie, à établir une zonation à valeur environnementale, et par la géoarchéologie, à insérer les perturbations anthropiques dans cet épais dépôt tout en dégageant les caractères microchronologiques des occupations.

L'étude palynologique réalisée correspond à l'ensemble sédimentaire inférieur de limons à granules dans lequel est intercalé un niveau riche en vestiges organiques carbonisés, précédant des li- 


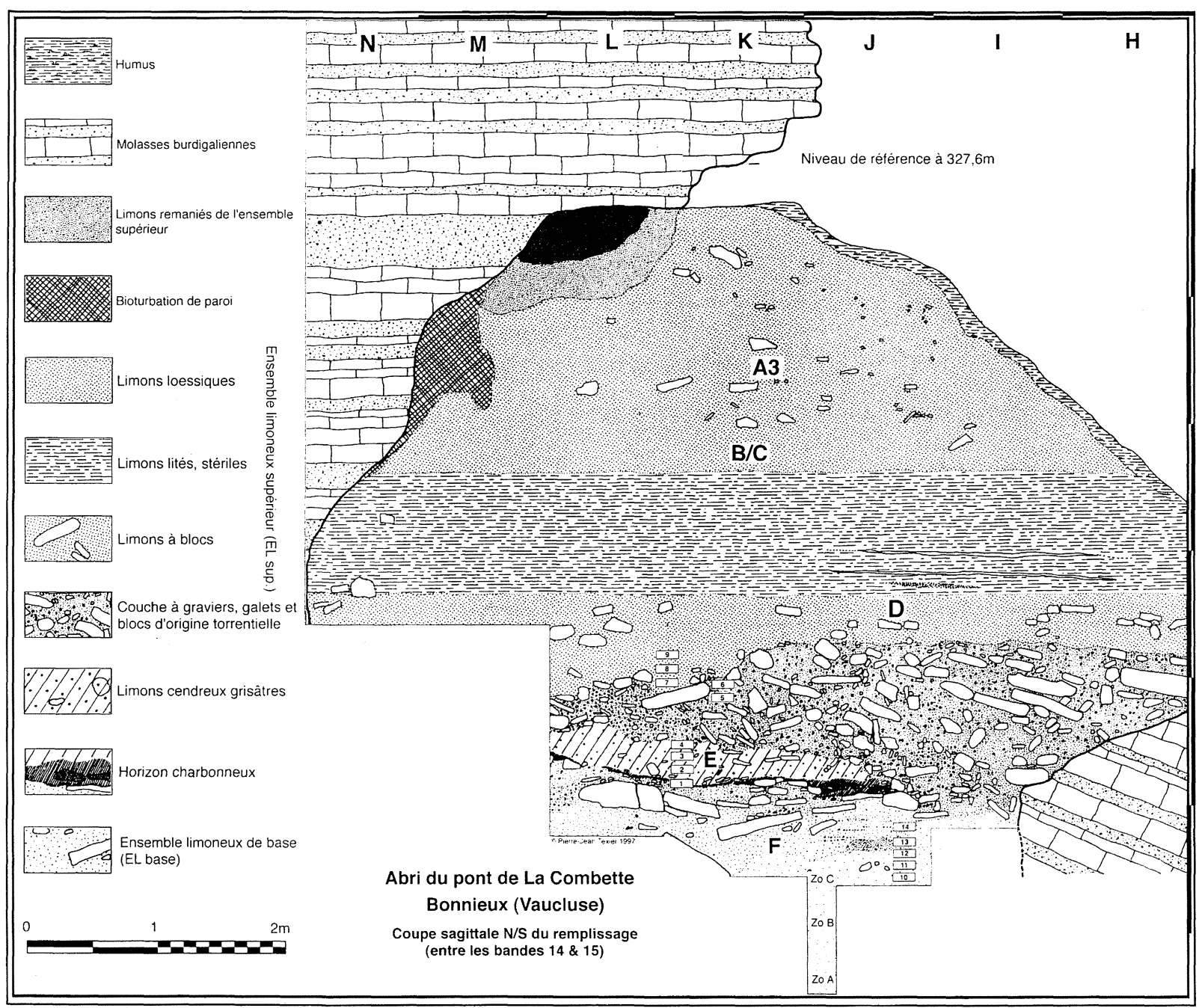

Fig. 2. Localisation du carottage 1995 dans la stratigraphie (Zo A, Zo B, Zo C) et emplacement des prélevements effectués à l'issue de la campagne de fouille 1997.

mons sableux un peu plus clairs avant ce qui pourrait être le substratum..

\section{CONTEXTE ARCHÉOLOGIQUE}

Cinq niveaux d'occupation ont pu à ce jour, être clairement individualisés dans ce remplissage sédimentaire. Les témoins archéologiques mis au jour dans l'ensemble limoneux supérieur (A, B/C et D) présentent globalement les mêmes caractéristiques et semblent nettement se différencier du contenu des niveaux inférieurs ( $E$ et $F$ ) actuellement en cours de fouille. Le niveau $\mathrm{D}$ situé à la base de l'ensemble limoneux supérieur qui a notamment pu faire l'objet d'un décapage extensif, a révélé une distribution hétérogène des objets, liée semble-t il à la présence de plusieurs structures de combustion situées en bordure de la surface protégée par le surplomb rocheux. D'une manière générale on peut dire que les ensembles lithiques mis au jour dans les limons supérieurs sont caractérisés par l'importation massive depuis la partie septentrionale du territoire fréquenté, de supports déjà débités, souvent retouchés en racloirs dont une petite partie est toujours de dimensions exceptionnelles. La composition a priori assez étonnante de ces ensembles lithiques est le reflet d'options prises dans un but particulier, que l'archéozoologie et analyse fonctionnelle ont permis de cerner. Les vestiges osseux essentiellement représentatifs de deux espèces, le cheval et le bouquetin, sont caractérisés par la rareté des extrémités d'os longs et respectivement par une 
sur-représentation ou une sous-représentation des restes céphaliques. L'action humaine manifeste sur de nombreux ossements, associe souvent des stries de silex à des marques de percussion. Une acquisition locale de ces gibiers lors de chasses relativement sélectives de type affût, a été proposée (Texier et alii, 1998b).

C'est parmi les grands éclats et les racloirs mis au jour sous la partie abritée des niveaux d'occupation que se trouvent les objets ayant enregistré et conservé les traces d'une utilisation particulière. L'analyse intégrée de l'ensemble des informations recueillies, montre de manière argumentée, qu'à coté d'activités de boucherie et de travail du bois, les petits groupes d'individus qui fréquentaient alors l'abri de La Combette pendant des périodes de quelques jours, se sont aussi livré à un travail complexe de préparation des peaux (Texier et alii, 1998a). Il ont ainsi laissé derrière eux le témoignage d'une exploitation active et organisée du milieu s'exprimant par une anticipation sur les contraintes imposées par celui-ci.

Les informations encore trop fragmentaires et incomplètes dont nous disposons sur les niveaux les plus anciens de l'abri (E et F), objet de cette étude palynologique, laissent toutefois apparaitre des comportements sensiblement différents (Texier et alii, 1998a,b). La densité des vestiges y est beaucoup plus importante. Des traces omniprésentes, cendres, pierres, silex, os brûlés et charbons par milliers, laissent supposer une utilisation sensiblement différente du feu et de l'espace domestique. Le cerf vient s'ajouter au bouquetin et au cheval parmi les espèces chassées dont les ossements ont été intensément fracturés. D'autre part, malgré un taux toujours très élevé de matériaux importés depuis le nord, la composante locale semble avoir tenu une part nettement plus importante dans l'approvisionnement en silex. La composition des ensembles lithiques mis au jour dans ces niveaux laisse également supposer des comportements techniques assez différents privilégiant notamment une méthodes de débitage. L'outillage retouché parmi lequel ne figure aucun de ces outils de dimensions exceptionnelles caractéristiques des niveaux de l'ensemble supérieur, est lui-même de proportions plus modestes. En l'absence, pour l'instant, d' analyse fonctionnelle, d'étude technologique et de remontages, son rapport aux éléments bruts de débitage, nettement plus défavorable doit être considéré comme l'indice d'activités, de comportements techniques et/ou d'exploitation du territoire différents.

\section{LA VÉGÉTATION ACTUELLE}

La chaînon anticlinal du Luberon, d'orientation E-O, s'étend sur une soixantaine de kilomètres pour $5 \mathrm{~km}$ de large, de Manosque à Cavaillon, où il vient s'ennoyer sous le Miocène du Vaucluse et la plaine du Rhône. L'édification de cette imposante barrière naturelle serait une conséquence directe de l'épisode d'âge messinien de la phase tectonique rhodanienne (Clauzon, 1974). Durant cet épisode tectonique, qui s'est déroulé il y a environ six millions d'années, se creusaient également les vallées synclinales d' Apt (Coulon-Calavon) et de la basse Durance. Dressé à la limite du domaine subalpin et du domaine provençal, l'écran montagneux du Luberon, d'une altitude moyenne de $800 \mathrm{~m}$, constituerait une barrière difficilement franchissable, n'était la percée en cluse des gorges de l'Aiguebrun entre le Grand et le Petit Luberon. L'abri de La Combette s'ouvre dans cet environnement géologique.

Initiée au Tertiaire, l'évolution karstique du paysage s'est intensifiée au cours des périodes les plus froides du Plio-Quaternaire. Elle s'est manifestée par une accentuation notable du creusement de canyons, le développement d'un karst profond et le colmatage des dépressions fermées (Jorda, 1992).

Directement ou indirectement, de nombreux paramètres sont conditionnés par le contexte géologique comme le relief et donc le gradient thermique et les hauteurs de précipitation, les voies de communications naturelles, le creusement d'abris de grottes et d'avens, etc. La végétation actuelle est logiquement le résultat direct de tout ces paramètres.

Le site de La Combette se situe à l'étage méditerranéen supérieur, caractérisé par la série du chêne vert (Quercetum ilicis) et du genévrier de Phoénicie (Juniperus phoenicea), remontant assez haut à la faveur d'escarpements calcaires bien exposés. Sont présents le chêne vert (Quercus ilex), la filaire (Phillyrea media), le fragon ou petit houx (Ruscus aculeatus), le chèvrefeuille implexe (Lonicera implexa) et le viorne tin (Viburnum tinus), mais sont aussi fréquents l' asperge à feuilles étroites (Asparagus acutifolius), le pistachier térébinthe (Pistacia terebinthus), le chèvrefeuille d'Etrurie (Lonicera etrusca), l'arbousier (Arbutus unedo), l'alaterne (Rhamnus alaternus), le chêne kermès (Quercus coccifera), la filaire à feuilles étroites (Phyllirea angustifolia), le buis (Buxus sempervirens) et le genévrier (Juniperus oxycedrus), qui forment un ensemble très caractéristique d'une végétation mé- 
diterranéenne (Molinier, 1938; Pons, 1950/1951).

D'autres communautés végétales remarquables du Luberon (Molinier, 1938; Pons, 1950/1951, 1951, 1952) apparaissant à proximité du site. On peut notamment citer Querceto pubescentis-Buxetum, la chênaie de chênes pubescents ou blancs (Quercus pubescens) associés à Buxus sempervirens, l'érable opale (Acer opalus), le cerisier de Sainte-Lucie (Prunus mahaleb), la mélisse (Melittis melissophyllum), la viorne lantane (Viburnum lantana) et le sorbier domestique ou cormier (Sorbus domestica) parmi les espèces plus caractéristiques. Le buis (Buxus sempervirens) confère à la végétation du Luberon une physionomie très particulière (Molinier, 1963), surtout en référence aux stades de dégradation de la chênaie pubescente. Le buis est également très abondant dans les taillis des chênes particulièrement autour du site. Le remplacement du chêne vert par le chêne pubescent étant progressif vers l'étage collinéen, les groupements sont nombreux et nettement plus forestiers que ceux de l'étage méditerranéen, en particulier dans le Grand Luberon.

La hêtraie à Fagus sylvatica n'est présente que dans le Grand Luberon dans sa partie la plus élevée. Il s'agit ici d'une formation végétale presque relictuelle (Mathon, 1951; Molinier, 1963; Pons, 1952).

Au niveau régional, cet espace constitue une zone refuge pour de nombreuses espèces de régions froides. L'ensemble présente une flore extrêmement riche et intéressante par le nombre des plantes rares présentes, la plupart venant de régions tempérées et froides. Ainsi peut ont noter la presence de: Sanguisorba officinalis, Lathraea squamaria, Phyllitis scolopendrium, Sanicula europaea, Taxus baccata, Hypericum hirsutum, Symphytum tuberosum, Primula elatior, Geranium pyrenaicum, Ribes alpinum, Ilex aquifolium et Circaea lutetia$n a$. Le Luberon fait donc preuve d'une grande originalité et d'un intérêt remarquable sur le plan de la diversité botanique. On estime en effet entre 1200 et 1500 , le nombre de taxons vasculaires qui s'y développent (Guende, 1993). La particularité de cette flore se signale tout d'abord par la présence d'un nombre important d'espèces rares d'intérêt national ou régional, ce qui est la preuve du maintien d'une qualité générale de l'environnement. Aussi peut-on également noter la présence d'espèces comme Ophrys bertolonii, Orchis fragans, Rosa gallica, Tulipa praecox, $T$. sylvestris et T. agenensis (tulipes sauvages devenues très rares en France), Ephedra nebrodensis ou Dictamnus albus. A ce titre, le Luberon se situe donc comme un carrefour biogéographique d'un véritable intérêt patrimonial, dû à sa grande diversité géologique avec des substrats variés, des conditions des reliefs contrastées, un réseau hydrographique important et des pratiques agricoles et forestières encore traditionelles (Guende, 1993). L'étude palynologique peut aider à comprendre beaucoup mieux le pourquoi de cette originalité floristique et aussi évaluer le niveau d'anthropisation du milieu pendant la période étudiée.

Un premier relevé botanique autour du site à permis d'identifier les espèces suivantes: Quercus ilex, Asparagus acutifolius, Thymus vulgaris, Ulmus glabra, Ilex aquifolium, Helychrysum stoechas, Buxus sempervirens, Hedera helix, Rubia peregrina, Cistus albidus, Salix cinerea, Ceterach officinarum, Pistacia terebinthus, Daphne laureola, Viola sylvestris, Asplenium trichomanes, Quercus pubescens, Rubus fruticosus, Hippophäe rhamnö̈des, Acer campestre, Acer monspeliensis, Cornus mas, Taraxacum officinalis, Juniperus communis, Corylus avellana et Medicago arborea. La végétation autour du site de La Combette s'avère donc assez particulière, offrant un mélange entre des éléments floristiques typiques de la végétation méditerranéenne (Quercetum ilicis) mais aussi de la forêt caducifoliée du Querceto pubescentisBuxetum, ainsi que d'élements floristiques pouvant être considérés comme reliques comme le houx (Ilex aquifolium), le châtaignier (Castanea sativa) ou le noyer (Juglans regia). On peut donc penser que le vallon de La Combette, par sa situation géographique exceptionelle dans la zone de transition entre le Petit et le Grand Luberon, a pu être un refuge pour différents éléments floristiques pendant le Pleistocene et l'Holocène, qui on ainsi pu subsister pendant les périodes froides du Quaternaire.

\section{MÉTHODOLOGIE}

L'échantillonage a été effectué à la tarière à main (tarière hollandaise) par l'un des auteurs (P. J. T.) au moment de la découverte de l'ensemble limoneux inférieur. La tarière a été soigneusement nettoyée entre chaque prélèvement. Sept échantillons on pu ainsi être recueillis sur un peu plus d'un mètre d'épaisseur dans la partie inférieure de la séquence stratigraphique (niveaux $\mathrm{F}, \mathrm{G}$ et $\mathrm{H}$ ).

Les sédiments ( 50 grs. par prélèvement) ont été traités par la méthode chimique classique qui met en jeu successivement l' acide chlorhydrique $(\mathrm{ClH})$,

T. P., 55, n. ${ }^{\circ} 2,1998$ 
l'acide fluorhydrique $(\mathrm{FH})$ et la potasse $(\mathrm{KOH})$. Une hyperconcentration des culots a été faite par lévigation du résidu en liqueur dense de Thoulet (Girard et Renault-Miskovsky, 1969).

L'identification des pollens et spores a été faite d'après les critères retenus parValdés et alii (1989), Moore et alii (1991) et Reille (1992, 1995). Le spectre pollinique de chaque échantillon n'a été établi qu'après le comptage d'un minimum de 150 grains et la reconnaissance d'une vingtaine de taxons différents (Mc Andrews et King, 1976; Janssen, 1981). L'identification des microfossiles nonpolliniques a été faite d'après les critères proposés parVan Geel et aili (1980/1981, 1983, 1984, 1989) et Pals et alii (1980). La courbe des pollens d'arbres (AP) par rapport aux pollens de plantes herbacées (NAP) constitue une première colonne du diagramme établi en pourcentages relatifs (Fig. 3).

La somme de base a été calculée par rapport aux taxons AP et NAP, et n'inclue pas les taxons hydro et hygrophiles ni les les fougères et microfossiles non-polliniques.

\section{RÉSULTATS ET DISCUSSION}

La sédimentation en grotte et sous abri, importante lors du dernier glaciaire, est responsable de l'enfouissement et de la conservation de nombreux sites moustériens dans le sud-est de la France. Ainsi les études portant sur l'environnement des Neándertaliens sont-elles assez nombreuses (Cattani et Renault-Miskovsky, 1989). Cependant, les séquences polliniques et climatiques sont difficiles à mettre en parallèle car nous ne disposons pas toujours d'éléments de datation satisfaisants pour affirmer leur contemporanéité (Leroi-Gourhan et RenaultMiskovsky, 1977).

Á La Combette, l'étude des macro et micromammifères de la partie supérieure du remplissage ainsi que celle des mollusques (Buisson-Catil, 1994), suggère un dépôt des sédiments lors d'une période fraîche voire froide du début du dernier glaciaire (début du stade isotopique 3). Au niveau pollinique, le diagramme qui ne porte pour l'instant que sur les couches les plus profondes, se situerait

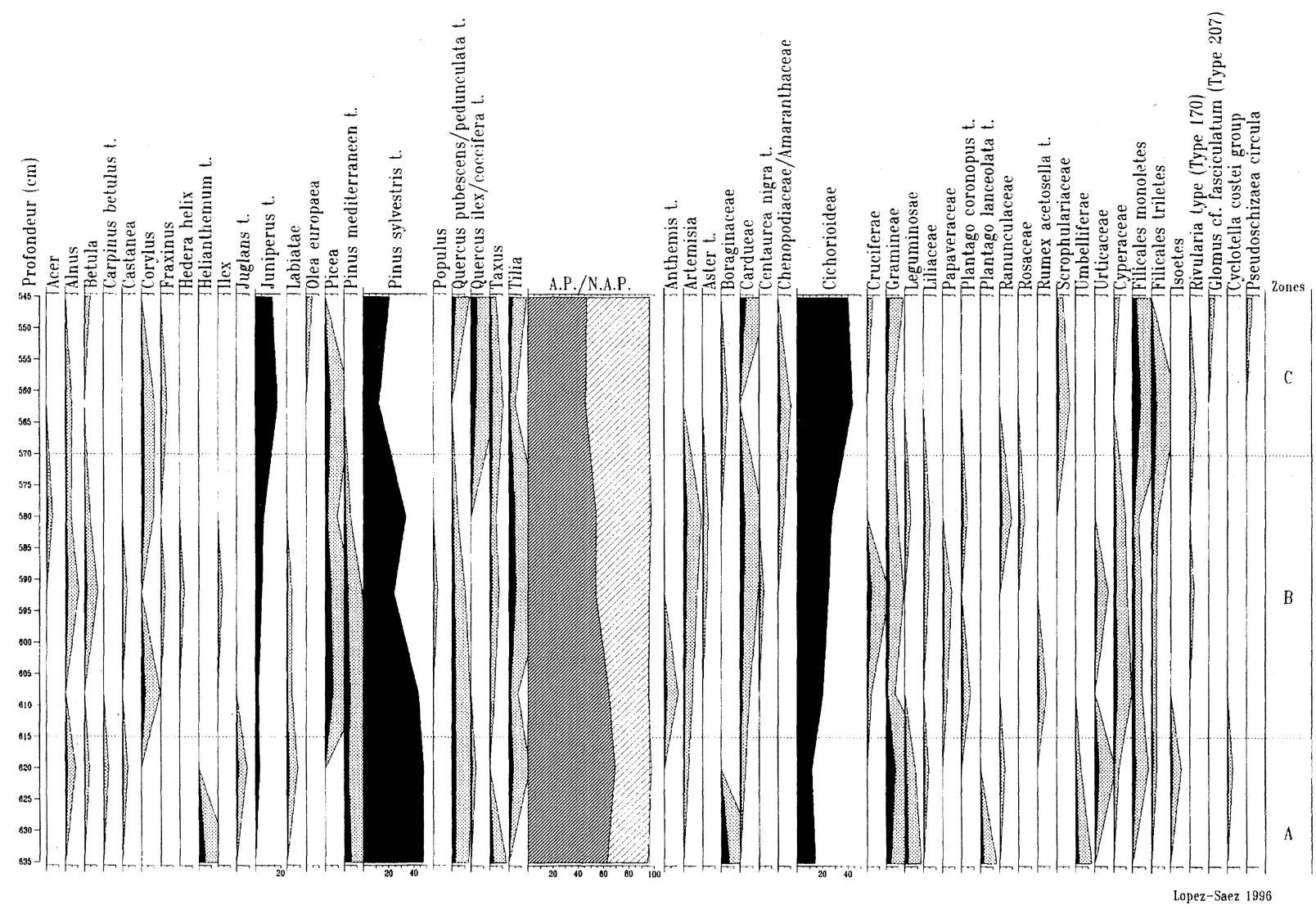

Fig. 3. Abri du pont de La Combette, Pléistocène superieur, dernier glaciaire, début du stade isotopique 3. Spectre pollinique de l'ensemble limoneux de base (EL base). 
dans le Würm ancien, probablement au niveau de l'interstade de Moershoofd, entre 50 et $60 \mathrm{ka} \mathrm{BP}$. Les mesures TL obtenues pour les limons supérieurs dont la moyenne est de $57400 \mathrm{BP}+/-4.3 \mathrm{ka}$ et que d'autres mesures en cours sur silex brûlés pourraient sensiblement rajeunir, sont en accord avec la chronologie palynologique proposée.

Pour une meilleure compréhension du diagramme pollinique (Fig. 3) celui-ci est subdivisé en trois biozones polliniques (A, B, C).

Le pin sylvestre (Pinus sylvestris t.) est le taxon dominant la végétation arborée dans la plupart des diagrammes, ce qui est aussi le cas ici, particulièrement dans les zones $\mathrm{A}$ et $\mathrm{B}$ et confirme l'âge würmien du sédiments (Leroi-Gourhan et RenaultMiskovsky, 1977). Cependant plusieurs oscillations climatiques tempérées semblent avoir interrompu cette période glaciaire. En effet deux phases plus thermiques (zones $\mathrm{A}$ et $\mathrm{C}$ ) viennent encadrer une phase typiquement steppique (zone B). Cette dynamique où l'alternance entre les phases froides et chaudes est très courte, est en accord avec la chronologie pollinique du site et se place dans le Würm ancien. La végétation arborée est presque toujours dominante tandis que la représentation des taxons steppiques reste très restreinte, ce qui fait référence à un climat encore relativement doux et humide durant cette phase du Würm ancien.

On peut dire de manière générale que la zone $\mathrm{A}$ du diagramme est dominée par la présence de Pinus sylvestris t. (47\%) ainsi que par celle des chênes caducifoliés (Quercus pedunculata/pubescens t., 2.6-3.3\%). La dominance du pin sylvestre indique un climat paraissant froid dans l'ensemble, mais marque par la présence de Carpinus betulus t. $(0.8 \%)$, Juglans t. $(1.7 \%)$ et Castanea $(0.8 \%)$ qui sont absents des niveaux supérieurs (zones $B$ et $\mathrm{C}$ ), cette zone paraît cependant nettement plus chaude que la suivante. La présence du charme (Carpinus betulus) est un bon indice d'une phase de réchauffement (Leroi-Gourhan, 1961). L'aulne (Alnus) avec $1.7 \%$ et les chênes à feuille caduque (Quercus pedunculata/pubescens $\mathrm{t}$.) sont aussi bien représentés. En concordance avec cette proposition on note aussi la présence de Quercus ilex-coccifera t. (0.8 $\%)$ et de Pinus méditerranéens t. (3.3-5.1\%)

La zone A serait donc une zone encore relativement froide mais un réchauffement progressif aurait cependant permis l'apparition du charme, du châtaignier et du noyer dans des endroits refuges, ainsi qu'un développement limité de la végétation du type méditerranéenne.
La relation A.P./N.A.P. est presque toujours de 60/40. Plus de $40 \%$ desA.P. sont des pollens de pin sylvestre. Avec des taux de pollen d'arbres relativement modestes ainsi que la présence d'espèces héliophiles (noisetier, bouleau, aulne), le paysage devait être celui d'une forêt très ouverte dominée par le pin tandis que les espèces mésothermophiles typiques des forêts tempérées se seraient réfugiées dans les vallons. En accord avec les donnés de la faune malacologique des limons supérieurs et limons moyens lités (Fig. 2), elle témoigne d'un environnement très ouvert (formations végétales à herbacées dominantes) et d'un climat froid (Magnin, 1991). L'ouverture des formations végétales est confirmée dans les niveaux supérieurs par la faune des micro-vertébres. Magnin (1991) note également que la présence de Chilostoma squamatinum (espèce sud-ouest-européene de milieux forestiers ouverts) sur le site s'expliquerait soit par la présence de micro-habitats favorables, le vallon de $\mathrm{La} \mathrm{Com}$ betté constituant alors un refuge, soit par la capacité adaptative de cette espèce à des conditions rigoureuses du Würm ancien. La présence d'espèces végétales mésotermophiles dans la zone $A$ ainsi que termophiles dans la zone $\mathrm{C}$ (Olea europaea) confirme le caractère refuge du vallon de La Combette pour la végétation et la faune pendant le pléniglaciaire. Seul le bassin méditerranéen semble avoir été une zone refuge pour les essences typiquement méditerranéennes qui apparaissent ou même se développent dans les parties les plus chaudes des diagrammes (Leroi-Gourhan et Renault-Miskovsky, 1977) ce qui est précisément le cas ici.

Guende (1993) note que l'on ne rencontre actuellement le châtaignier (Castanea sativa) que rarement en Luberon. Il est alors dispersé dans des fonds de vallons aux sols profonds et frais où il trouve les meilleures conditions de développement, c'est à dire dans des expositions écologiques refuges. Ce caractère relictuel, valable également pour Juglans, a été confirmé par la palynologie en Espagne et en France (Renault-Miskovsky et alii, 1984; García Antón et alii, 1990). La présence du noyer est rare mais il a été cité dans de nombreux diagrammes d'Europe occidentale de la base du Pléistocene à la fin du Paléolitique moyen (Leroi-Gourhan et Renault-Miskovsky, 1977). On peut donc considérer ces deux taxons comme des espèces reliques au sein du site de La Combette.

Pour la région méditerranéenne, il est apparaît évident que le Vaucluse constitue une limite septentrionale. Cette limite est particulièrement nette avec

T. P., 55, n. ${ }^{\circ} 2,1998$ 
le Mont Ventoux qui forme une barrière vigoureuse (Girerd, 1977/1978). Mais la notion de région méditerranéenne étant variable suivant les critères retenus, les massifs montagneux vauclusiens en sont parfois exclus. La région méditerranéenne se définit classiquement par la limite de l'olivier, mais peut aussi bien être définie par la limite du chêne vert, ou tout au moins par son association, suivant la méthode phytosociologique de Braun-Blanquet. Par contre, si les limites sont basées sur des critères climatiques, la totalité du département y est alors incluse. Cette dernière façon de définir la région méditerranéenne explique que l'on peut constater l'influence méditerranéenne dans tous les étages de végétation du Vaucluse (Girerd, 1977/1978) et particulièrement en Luberon. D'un point de vue paléoécologique, l'ensemble de taxons identifiés par les pollens est représentatif d'une forêt tempérée. On notera cependant la présence de pollens d'olivier (Olea europaea) dans la zone C, mais aussi de Quercus ilex-coccifera $\mathrm{t}$. dans les zones A et $\mathrm{C}$, de Pinus méditerranéennes $\mathrm{t}$. dans tout le diagramme, sans oublier Castanea et Juglans t. dont nous avons déjà fait état. Il s'agit d'éléments floristiques typiques d'une végétation méditerranéenne peu développée pendant la période étudiée mais présents par contre à proximité du site. Les exemples ne sont pas rares pour le Pléistocène moyen et inférieur du sud de la France d'épisodes durant lesquels a été mise en évidence la présence de taxons méditerranéens en proportions non négligeables (Beaulieu, 1967; Leroi-Gourhan et Renault-Miskovsky, 1977; Renault-Miskovsky et Girard, 1988; Renault-Miskovsky et alii, 1992). On peut dont supposer que la végétation du site a presque toujours été pendant la période étudiée, de type froid à tempéré, avec une forte représentation des espèces arborées. Les taxons méditerranéens étaient certainement bien représentés dans l'ensemble régional et très probablement aux abords du gisement.

La présence de Taxus $(2.6 \%)$ dans le début de la zone A mais pas après, est un bon indicateur d'une première phase plus froide (subzone A-1) avec l'if et Helianthemum t. (5.1\%) et d'un réchauffement progressif où l'if a presque disparu (subzone A-2). On note en revanche, l'apparition du charme, du châtaignier et du noyer. Le réchauffement est accompagné d'une augmentation de l'humidité et le développement des fougères monolètes $(0$ à $2.5 \%)$ et triletes $(0$ à $0.8 \%)$, ainsi que d'Isoetes $(1.7 \%)$ et de Cyperaceae $(0.8 \%)$, indicateurs d'une augmentation de l'humidité édaphique et ambiante.
Une diatomée, Cyclotella costei group, a été trouvée en zone $\mathrm{A}$. Cette présence, même à un seul exemplaire, est indicatrice d'un milieu aquatique peu profond. Les diatomées sont de bons indicateurs d'une humidité ambiante assez élevée ainsi que de l'existence de sources d'eau douce (Brochier et Druart, 1993).

La zone $\mathrm{B}$ suggère par contre, le développement d'un épisode steppique avec le développement significatif d'Artemisia (1.4 à $2.8 \%$ ) ainsi que d'autres taxons steppiques comme Centaurea nigra t., Cruciferae (3.5\% maximum) et Papaveraceae (1.4 $\%$ maximum).

Toujours dans cette zone B, on note la disparition du noyer, du chêne vert et du charme, ainsi que le caractère relictuel du châtaignier. Alnus, Fraxinus et Populus sont également présents comme arbres indices de l'existence d'une ripisylve. On notera que l'existence d'une ripisylve est également suggérée dans, les niveaux supérieurs, par la présence d'Arvicola terrestris (Magnin, 1991).

Le refroidissement enregistré en zone B permet un nouveau développement de l'if (Taxus), ainsi que la présence constante de l'épicea (Picea abies), qui commençait déjà à apparaître à la fin de la zone A. On note également l'unique apparition du houx (Ilex). La zone B témoigne donc d'une phase plus froide et steppique, avec une petite montée de la végétation herbacée.

L'épicéa (Picea abies) est bien représenté dans le diagramme (1.9 à $5.8 \%$ ), mais en particulier, au tout début de la zone B. Leroi-Gourhan (1961) indique que son pollen se retrouve presque partout jusqu'au dernier interglaciaire mais également pendant le Würm. L'épicéa a donc subsisté en certains point de l'est et du sud-est de la France durant cette période. Ceci vient confirmer le caractère de refuge botanique de l'environnement immédiat du site.

Il est par ailleurs assez surprenant, pendant cette phase steppique, de constater la persistance du châtaignier $(0.7 \%)$ et des pourcentages encore assez élevés de Pinus méditerranéens t. (0.9 à $4.3 \%$ ), qui ne peuvent s'expliquer que par le caractère relictuel de la végétation du vallon ou par la présence des formations méditerranéenes assez éloignées du site, en un lieu où le climat devait être moins rigoureux.

I. Thery a mis en évidence dans la couche $\mathrm{E}$ (Thery, 1998), une très forte proportion de charbons de Pinus sylvestris/nigra mais signale aussi la présence de Buxus et d'Acer. L'analyse pollinique des dépôts würmiens a montré que l'association à pins sylvestres s'est développée à la faveur de conditions 
de type interglaciaire au cours desquelles le pin a colonisé les vastes espaces découverts mis en place pendant les phases glaciaires. Le pin sylvestre est une espèce peu exigeante, souvent pionnière, colonisatrice d'espaces vides. Ainsi occupe t-il des stations ou d'autres essences ne peuvent pousser en premier (Ozenda, 1985). Betula a parfois un comportement similaire, en particulier dans l'étage montagnard. L'étude anthracologique réalisée confirme la présence dominante du pin de type sylvestris/nigra à proximité de l'abri (Thery, 1998) et permet d'évoquer des conditions climatiques plus froides que l'actuel mais un couvert végétal néanmoins bien développé.

Le taux des pollens de pin sylvestre passe en dessous de $40 \%$ dans la zone $\mathrm{B}$ et à moins de $30 \%$ dans la zone A. Deux facteurs peuvent expliquer cette évolution de la courbe du pin sylvestre: en premier lieu, l'évolution d'une végétation steppique avec la montée des espèces herbacées et d'artemisia en particulier; en second lieu, l'augmentation progressive de l'anthropisation du milieu, reflétée par la montée progressive des Cichorioideae depuis la zone $\mathrm{A}(11.7-14.1 \%)$, se poursuivant dans la zone B (20.3-27.1\%), pour atteindre son maximum en zone $C(40.2-43.8 \%$ ), où le pourcentage du pin sylvestre chute jusqu'à $12.5 \%$.

Le tilleul (Tilia), présent dans tout le diagramme, est plus fréquent dans les stations interglaciaires et pendant les interstades würmiens (Leroi-Gourhan, 1961). Sa présence dans les sédiments de La Combette évoque clairement un interstade chaud du Würm ancien. Tilia platyphyllos (Guende, 1993) est l'espèce actuellement connue dans le Luberon.

Le taux du noisetier (Corylus) est peu élevé (1.9$2.9 \%$ ) mais Triat (1971) a mis en évidence la faible représentation pollinique de ce taxon au sein des corylaies dans leQuercetum pubescentis du Grand Luberon. Le noisetier, espèce pionnière favorisée par l'ouverture de la forêt et l'existence d'espaces vides où il peut s'installer, est en revanche un bon indicateur des phases intermédiaires entre réchauffement et refroidissement. La présence d'espèces héliophiles'(Corylus, Betula, Alnus) en pourcentages plus importants dans la zone B, confirme l'ouverture de la forêt et l'installation de conditions steppiques.

Enfin, la zone $\mathrm{C}$ voit pratiquement disparaître Artemisia, ainsi que le houx (Ilex). Cette zone est surtout caractérisée par l'apparition de l'olivier (Olea europaea), le progrès du genévrier (Juniperus t.) ainsi que par des forts pourcentages de $\mathrm{Ci}$ chorioideae.
$\mathrm{Au}$ point de vue climatique c'est encore une phase froide avec Pinus sylvestris t. (12.5-20.6\%), Picea $(4.2 \%)$ et Taxus (0.9-2.1\%), mais pas vraiment steppique. L'anthropisation maximum du milieu est clairement marquée par la montée de la courbe des Cichorioideae jusqu'à le $40 \%$ du total, accompagnée par d'autres taxons nitrophiles comme Boraginaceae, Carduaceae, Chenopodiaceael Amaranthaceae. L'anthropisation du milieu était déjà manifeste dans les autres zones mais moins marquée que dans la zone C. A faveur de cette influence de l'homme sur le milieu ont note également l'apparition de deux microfossiles non-polliniques indicateurs des phases d'érosion et de sécheresse: ce sont Glomus cf. fasciculatum et Pseudoschizaea circula (= Concentrycistes). Le Type 170 (Rivularia type) correspond à une Cyanobacterie, indicatrice d'eaux profondes et non-stagnantes ainsi que des milieux méso-oligotrophiques (Van Geel et alii, 1983). Cette cyanobacterie est capable de fixer le nitrogène et peut vivre dans des milieux pauvres en nitrogène ou oligotrophiques, ce qui semble être le cas dans la zone $C$.

La zone $\mathrm{C}$ montre clairement la première apparition du chêne vert (Quercus ilex-coccifera t.) ainsi que de l'olivier (Olea europaea). Ces taxons termophiles ont probablement perduré dans des zones refuges (Cattani et Renault-Miskovsky, 1989) mais on peut aussi penser à l'existence de formations méditerranéennes loin du site, où le chêne vert serait alors dominant sur le pin méditerranéen qui a pratiquement disparu à la zone $\mathrm{C}$. On rencontre également le pollen d'Olea ainsi que celui de Betula dans les niveaux supérieurs de La Combette, ce qui confirme son caractère relictuel dans le site pendant le Pléistocène supérieur.

La zone C a enregistré l'existence d'une phase plus sèche, encore froide mais pas steppique, caractérisée par le développement du chêne vert et de l'olivier. L'absence du charme peut indiquer l'absence d'un réchauffement pendant cette phase C. La baisse des pourcentages de pin sylvestre peut s'expliquer soit par l'anthropisation du milieu soit par la sécheresse. Le genévrier (Juniperus t.) serait favorisé par l'existence d'espaces ouverts consécutivement au développement de la végétation steppique évoqué par la zone B. Il jouerait le rôle d'une espèce pionnière et colonisatrice, particulièrement à faveur de la sécheresse régnant cette époque. Son taux atteint $17.7 \%$ dans la zone $C$.

En conclusion, on peut dire que l'ensemble des espèces de la paleovégétation identifiée dans les

T. P., 55, n. $^{\circ} 2,1998$ 
niveaux inférieurs du site de La Combette, est globalement représentative de milieux froids à tempérés. Certains taxons, dont les taxons méditerranéens, ont trouvé refuge dans le vallon de La Combette pour perdurer pendant les périodes les plus froides de la dernière glaciation. Plusieurs phases climatiques, successivement fraîche et humide (subzone A-1), tempéré et humide (subzone A-2), froide et steppique ou sèche continentale (zone $\mathrm{B}$ ) et froide et sèche (zone $\mathrm{C}$ ) permettent néanmoins l'implantation d'une forêt bien développée dominée par les pins sylvestres; la forêt caducifoliée (avec le charme, le noyer, le châtaignier, le chêne pubescens/pédonculé, le noisetier, etc) se réduit à des bouquets d' arbres dispersés ou bien se réfugient dans des vallons ou ripisylves étroits.

La succession des forêts s'est effectuée à partir d'une pinède à espèces mésothermophiles (zone A), à laquelle a succédé une steppe-prairie à pin sylvestre (zone B) puis une prairie boisée à pin sylvestre, et à espèces caducifoliées et thermophiles (zone C). Cette dynamique de la végétation ressemble beaucoup à celle exposée dans les synthèses de Cattani et Renault-Miskovsky (1989) et Renault-Miskovsky (1972), prenant en compte des sites correspondant probablement au mêmes périodes. Il n'existe aucune formation lacustre ou tourbeuse en Vaucluse suffisamment ancienne, pour avoir une idée précise de la végétation au Würm ancien. Les grandes séquences polliniques continues de France comme Les Echets (Beaulieu et Reille, 1984) ou la Grande Pile (Woillard, 1978) sont malheureusement plus anciennes que celle concernée par notre étude. L'analyse pollinique du site de La Combette constitue donc un apport intéressant pour appréhender la paléovégétation au début du stade isotopique 3 , lors du dernier épisode glaciaire.

\section{BIBLIOGRAPHIE}

Abassi, M. et Desclaux, E. (1996): “Arvicola Lacepede, 1799 (Rodentia Mammalia) de quatre séquences du sudest de la France et de Ligurie datant de la fin du Pleistocène moyen et du Pleistocène supérieur". Quaternaire, 7 (1): 29-37.

Beaulieu DE, J.L. (1967): “Aspects de la végétation d'un nouveau site préhistorique mindélien des Alpes-Maritimes, d'après l'analyse pollinique". Comptes Rendus de l'Academie des Sciences, 264. Série D: 2741-2743.

Beaulieu DE, J.L. et Reille, M. (1984): “A long Upper Pleistocene pollen record from les Echets, near Lyon, France". Boreas, 13: 111-132.

Brochier, J.E. (1976): "Abri de la Combette". En Livret-
Guide de l'excursion C2 "Provence et Languedoc méditerranéen, sites paléolitiques et néolitiques”. CRNS: 113. Nice.

- (1977): "Évolution des climats et des paysages vauclusiens au cours du Würmien récent et du Postglaciaire". Paleoécologie de l'homme fossile, 6: 1-180.

Brochier, J.L. et Druart, J.C. (1993): "Le milieu lacustre". Documents d'Archéologie Française, 40: 39-60.

Buisson-Catil, J. (ed.) (1994): "Le Paléolithique moyen en Vaucluse. A la rencontre des chasseurs néandertaliens de Provence nord-occidentale". Notices d'archéologie vauclusienne, 3: 1-142.

- (1997): "Le Luberon des origines". Notices d'archéologie vauclusienne, 4: 1-62.

Cattani, L. et Renault-Miskovsky, J. (1989): "La réponse des végétations aux variations climatiques quaternaires autour des sites archéologiques du sud de la France et nord-est de l'Italie". Il Quaternario, 2: 147-170.

Clauzon, G. (1974): "Quel âge le Luberon a-t-il?". Etudes vauclusiennes, janv.-juin: 1-16.

García Antón, M.; Morla Juaristi, C. et Sainz Ollero, H. (1990): "Consideraciones sobre la presencia de algunos vegetales relictos terciarios en el Cuaternario de la Península Ibérica". Boletín de la Real Sociedad Española de Historia Natural (Sección Biología), 86: 95-105.

Geneste, J.M. (1989): "Economie des ressources lithiques dans le moustérien du soud-ouest de la France". En M. Otte (ed.): L'Homme de Néandertal. Actes du colloque international de Liège (4-7 décembre 1986). En J.K. Kozlowski (coord.): La subsistance, 6. Service de Prehistoire. Université de Liège: 75-97.

Girard, M. et Renault-Miskovsky, J. (1969): “Nouvelles techniques de préparation en palynologie appliquées à trois sédiments du Quaternaire final de l"abri Cornille (Istres, Bouches-du-Rhône)". Bulletin de la Association Française pour l'Éude du Quaternaire, 4: 275-284.

Girerd, B. (1977/1978): "Inventaire écologique et biogéographique de la flore du departement de Vaucluse". Bulletin de la Société d'Études Naturelles de Vaucluse, 47/48: 1-347.

GuEnde, G. (1993): Flore du Luberon. Édisud. Aix-en-Provence.

JANSSEN, C. (1981): "Contemporary pollen assemblages from the Vosges (France)". Review of Palaeobotany and Palynology, 33: 183-313.

JORDA, M. (1992): "Les monts de Vaucluse et le plateau d'Albion. Paysages, géomorphologie, évolution quaternaire". Alpes de Lumières, 11: 8-13.

LEMORINI, C. (1997): L'organisation du geste des néandertaliens. Analyse fonctionelle des industries lithiques de Grotta Breuil (Latium, Italia) et de La Combette (Bonnieux, Vaucluse, France). Doctorat Rijksuniversiteit Leiden. Leiden.

Leroi-Gourhan, A. (1961): "Flores quaternaires françaises". Bulletin de la Société Botanique de la France, 108: 244-254. 
Leroi-Gourhan, A. et Renault-Miskovsky, J. (1977): "La palynologie appliquée à l'archéologie: méthodes, limites et résultats". Bulletin de la Association Française pour l'Éude du Quaternaire, 47 (suppl.): 35-49.

MaGnin, F. (1991): Mollusques continentaux et histoire quaternaire des milieux méditerranéens (Sud-Est de la France, Catalogne). Thèse de Doctorat. UniversitéAixMarseille II. Aix-en-Provence.

MATHON, C. (1951): "Contribution à l'étude phytogéographique de la Haute-Provence occidentale. Important aspect méconnu de la Hêtraie du Luberon". Bulletin de la Société Botanique de la France,98: 58-67.

McAndrews, J.H. et King, J.E. (1976): "Pollen of the Noth American Quaternary: the top twenty". Geoscience and Man, 15: 41-49.

Molinier, R. (1938): "La végétation du Massif du Luberon (Vaucluse)". Bulletin Le Chêne, 45: 1-95.

Molinier, R. (1963): "Vue d'ensemble sur la végétation du Grand et du Petit Luberon (Vaucluse)". Bulletin du Musée d'Histoire Naturelle de Marseille, 23: 1-28.

Moore, P.D.; Webb, J.A. et Collinson, M.E. (1991): Pollen Analysis. Blackwell Scientific Publications. London. $2^{\text {nd }}$. Edition.

OzEnd, P. (1985): La végétation de la chaîne alpine dans l'espace montagnard européen. Ed. Masson. Paris.

Pals, J.P.; Van Geel, B. et Delfos, A. (1980): "Palaeoecological studies in the Klokkeweel bog near Hoogkarspel (Prov. of Noord-Holland)". Review of Palaeobotany and Palynology, 30: 371-418.

Pons, A. (1950/1951): "Observations sur quelques associations végétales du Massif du Grand Luberon (Vaucluse)". Bulletin de la Société d'Etudes des Sciences Naturelles de Vaucluse, 21-22: 1-22.

- (1951): "La flore de la chaîne du Grand Luberon". Bulletin duMusée d'Histoire Naturelle de Marseille, 11:15.

- (1952): "La hêtraie du Grand Luberon (Vaucluse)". Bulletin de la Société Botanique Française, 99: 7-18.

Reille, M. (1992): Pollen et spores d'Europe et d'Afrique $d u$ Nord. Laboratoire de Botanique Historique et Palynologie. Marseille.

- (1995): Pollen et spores d'Europe et d'Afrique du Nord, supplement 1. Laboratoire de Botanique Historique et Palynologie. Marseille.

Renault-Miskovsky, J. (1972): "Contribution à la paléoclimatologie du Midi Méditerranéen pendant la dernière glaciation et le Post-glaciaire, d'après l'étude palynologique du remplissage des grottes et abris sous roche". Bulletin du Musée d'Anthropologie Préhistorique de Monaco, 18: 145-210.

Renault-Miskovsky, J.; Bui-Thi-Mai et Girard, M. (1984): "A propos de l'indigenat ou de l'introduction de $J u$ glans et Platanus dans l'Ouest de l'Europe au Quaternaire". Revue de Paléobiologie, vol. spéc.: 155-178.

Renault-Miskovsky, J. et Girard, M. 1988. "Palynologie du remplissage de la grotte du Vallonet (Roquebrune-
Cap-Martin, Alpes-Maritimes). Nouvelles données chronologiques et paléoclimatiques". L'Anthropologie, 92: 437-448.

Renault-Miskovsky, J.; Arobba, D.; Audouze, F.; Barbero, M.; Bui-Thi-Mai, M.; Ducatillion, C.; Giacobini, G.; Girard, M.; Goudet, M.; Lumley, H.; Moulle P.E.; Sandoz, H. et Simone, S. (1992): "La végétation actuelle et la préhistoire de la Côte d'Azur et de la Riviera". Cahiers de Micropaléontologie, 7: 187-213.

Richter, D.; LANG, A. et WAGNER, G. (1996): “Luminescence dating in Archaeology". XII International Congress of Prehistoric and Protohistoric Sciences. Coloquia 2. Archaeometry: 9-15. Forli.

Texier, P.J.; Lemorini, C.; Brugal, J.P. et Wilson, L. (1998a): "Une activité de traitement des peaux dans l'habitat moustérien de La Combette (Bonnieux, Vaucluse, France)". Quaternaria Nova, 189-211.

TEXIER, P.J.; Lemorini, C.; BRUgal, J.P. et Wilson, L. (s.p.): "Fonction d'un site du Paléolithique moyen en marge d'un territoire: l'abri de La Combette (Bonnieux, Vaucluse)". Actes XVIIIe Rencontres Internationales d'Archéologie et d'Histoire d'Antibes: Economie Préhistorique, les comportements de subsistance au Paléolitique (Antibes, 1997).

THERY, I. (1998): Economie du combustible et paléoécologie en contexte glaciaire et périglaciaire, paléolithique moyen et supérieur du sud de la France (anthracologie, expérimentation, taphonomie). Thèse Université de Paris I. Paris.

TRIAT, H. (1971): "Contribution à l'étude des fréquences polliniques de Corylus aveliana L., corylaies du Luberon". Pollen et Spores, 13: 405-414.

VAldés, B.; Díez, M.J. et Fernández, I. (1989): Atlas polínico de Andalucía Occidental. Intituto de Desarrollo Regional, 43. Excma. Diputación de Cádiz.

VAN GeEl, B.; Bohncke, S.J.P. et Dee, H. (1980/1981): “A palaeoecological study of an Upper Late Glacial and Holocene sequence from De Borchert, The Netherlands". Review of Palaeobotany and Palynology, 31:367-448.

VAN GeEl, B.; CoOpe, G.R. et VAN DER Hammen, T. (1989): "Palaeoecology and stratigraphy of the Lateglacial type section at Usselo (The Netherlands)". Review of Palaeobotany and Palynology, 60: 25-129.

Van Geel, B.; DeLange, L. et WIEgers, J. (1984): “Reconstruction and interpretation of the local vegetational succession of a Lateglacial deposit from Usselo(The Netherlands), based on the analysis of micro-and macrofossil". Acta Botanica Neerlandica, 33: 535-546.

Van Geel, B.; Hallewas, D.P. et Pals, J.P. (1983): “A Late Holocene deposit under the Westfriese Zeedijk near Enkhuizen (Prov. of Noord-Holland, The Netherlands): Palaeoecological and archaeological aspects". Review of Palaeobotany and Palynology, 38: 269-335.

Woillard, G. (1978): "Grande Pile peat bog: a continous pollen record for the last 140.000 years". Quaternary Research, 9: 1-21.

T. P., 55, n. $^{\circ} 2,1998$ 